\title{
Scar less: a review of methods of scar reduction at sites of peripheral nerve repair
}

\begin{abstract}
Scar formation is important for normal wound healing but proliferation of scar tissue from the epineurium in response to a nerve injury will result in impediment to the regenerating axons that need to traverse the repaired site. This article reviews the events that occur after peripheral nerve transection and the challenges that need to be addressed to reduce scarring in order to improve nerve regeneration. (Oral Surg Oral Med Oral Pathol Oral Radiol Endod 2010; 109: 357-366)
\end{abstract}

\begin{tabular}{l|l|}
\hline Authors: & Ngeow, W. C. \\
\hline Journal: & $\begin{array}{l}\text { Oral Surgery Oral Medicine Oral Pathology Oral Radiology and } \\
\text { Endodontology }\end{array}$ \\
\hline Year: & 2011 \\
\hline Pages: & $357-366$ \\
\hline DOI: & $10.1016 /$ j.tripleo.2009.06.030 \\
\hline
\end{tabular}

\section{Keywords :}

RAT SCIATIC-NERVE; IMMUNE-MEDIATED DEMYELINATION; EPINEURAL SLEEVE NEURORRHAPHY; WALLERIAN DEGENERATION; SCHWANN-CELLS; HYALURONIC-ACID; FIBRIN GLUE; TRANSFORMING GROWTH-FACTOR-BETA-1; FUNCTIONAL RECOVERY; SURGICAL TECHNIQUE 


\section{Please cite as :}

NGEOW, W. C. 2010. Scar less: a review of methods of scar reduction at sites of peripheral nerve repair. Oral Surgery Oral Medicine Oral Pathology Oral Radiology and Endodontology, 109, 357-366.

\section{URL :}

- http://apps.webofknowledge.com/InboundService.do?SID=R13MkBKIBC2Ng\%40m6 3IE \&product $=$ WOS \&UT $=000274956600008 \&$ Src App $=$ CR \&DestFail $=$ http $\% 3 \mathrm{~A} \% 2 \mathrm{~F}$ \%2Fwww.webofknowledge.com\&Init=Yes\&action=retrieve\&Func=Frame\&customer $\underline{\text { sID }=\text { RID \&SrcAuth=RID \&IsProductCode }=\text { Yes } \& \text { mode=FullRecord }}$

- http://www.ncbi.nlm.nih.gov/pubmed/20219599

- http://www.mendeley.com/research/scar-less-review-methods-scar-reduction-sitesperipheral-nerve-repair-14/

- http://www.sciencedirect.com/science/article/pii/S1079210409004296

- http://cat.inist.fr/?aModele=afficheN\&cpsidt=22504159

- http://d.wanfangdata.com.cn/NSTLQK_10.1016-j.tripleo.2009.06.030.aspx 